\title{
A Comparison of Ballistic and Nonballistic Lower-Body Resistance Exercise and the Methods Used to Identify Their Positive Lifting Phases
}

\author{
Jason Lake, Mike Lauder, Neal Smith, and Kathleen Shorter \\ University of Chichester
}

\begin{abstract}
This study compared differences between ballistic jump squat (B) and nonballistic back squat (NB) force, velocity, power, and relative acceleration duration, and the effect that the method used to identify the positive lifting phase had on these parameters. Ground reaction force and barbell kinematics were recorded from 30 resistance trained men during B and NB performance with 45\% 1RM. Force, velocity, and power was averaged over positive lifting phases identified using the traditional peak barbell displacement (PD) and positive impulse method. No significant differences were found between B and NB mean force, and mean power, but B mean velocity was $14 \%$ greater than the NB equivalent. Positive impulse mean force was $24 \%$ greater than PD mean force, and B relative acceleration duration was $8.6 \%$ greater than the NB equivalent when PD was used to identify the end of the positive lifting phase. These results challenge common perceptions of B superiority for power development.
\end{abstract}

Keywords: biomechanics, force plate, kinematics, kinetics, strength, impulse

Ballistic resistance exercise is preferred for power development because resistance is accelerated for longer, and mean force, velocity, and power are greater compared with nonballistic resistance exercise (Hori et al., 2008; Newton et al., 1996). However, investigators have demonstrated that differences between ballistic and nonballistic resistance exercise occur largely because of the way in which the positive lifting phase is determined (Frost et al., 2008).

Nonballistic resistance exercise consists of distinct propulsion and braking phases (Gonzalez-Badillo \& Sanchez-Medina, 2010; Jandacka \& Vaverka, 2008; Sanchez-Medina et al., 2010). The propulsion phase can be identified by studying the velocity-time curve of the mass of interest and locating the period between transition from negative to positive velocity to peak velocity-points that correspond with zero net force. For the purpose of this study positive lifting phases that only consider the propulsion phase will be operationally defined as the positive impulse (PI) positive lifting phase. The braking phase which is fundamental to nonballistic resistance exercise performed through fixed ranges of motion can be identified by locating the period on the velocity-time curve between peak velocity and the transition from positive to negative velocity (peak displacement). For the

Jason Lake (Corresponding Author), Mike Lauder, Neal Smith, and Kathleen Shorter are with the Department of Sport and Exercise Science, University of Chichester, Chichester, West Sussex, U.K. purpose of this study positive lifting phases that consider both the propulsion and braking phase will be operationally defined as the peak displacement (PD) positive lifting phase. Inclusion of the braking phase exaggerates positive lifting phase duration, reducing measures of force, velocity and power averaged over the positive lifting phase, and relative acceleration duration - the basis of ballistic exercise preference; investigators apparently operate on the assumption that ballistic resistance exercise does not include a braking phase (Frost et al., 2008).

Investigators recently suggested that the braking phase should not be included in ballistic and nonballistic resistance exercise comparison because it is not common to both exercise types (Frost et al., 2008). This alternative method enables greater accuracy regarding the mechanical demands of nonballistic resistance exercise, but must be refined.

The propulsion phase of ballistic and nonballistic resistance exercise can also be derived from positive impulse; the product of positive net force multiplied by time, which is proportional to the resistance's change in momentum (Dowling and Vamos, 1993; Driss et al., 2001; Harman et al., 1991, Linthorne, 2001). Net force is obtained by subtracting barbell and body system weight from force (Kawamori et al., 2005). However, in the original application of this method, Frost et al. (2008; Figure 1, p. 373) identified positive impulse from absolute force (force including system weight), violating the correct application of basic mechanical principles (Driss et al., 2001; Linthorne, 2001). The traditional and alternative 
approach (applied to lower-body exercise) is illustrated in Figure 1 and 2. The approach described by Frost et al. (2008; adapted for ballistic lower-body exercise) is illustrated in Figure 3. They show the methodologically correct way of identifying the end point of the positive lifting phase form peak displacement (point c) and positive impulse (point $b$; the point at which net force decreases to zero), and a third (point $b^{*}$ ) caused by a

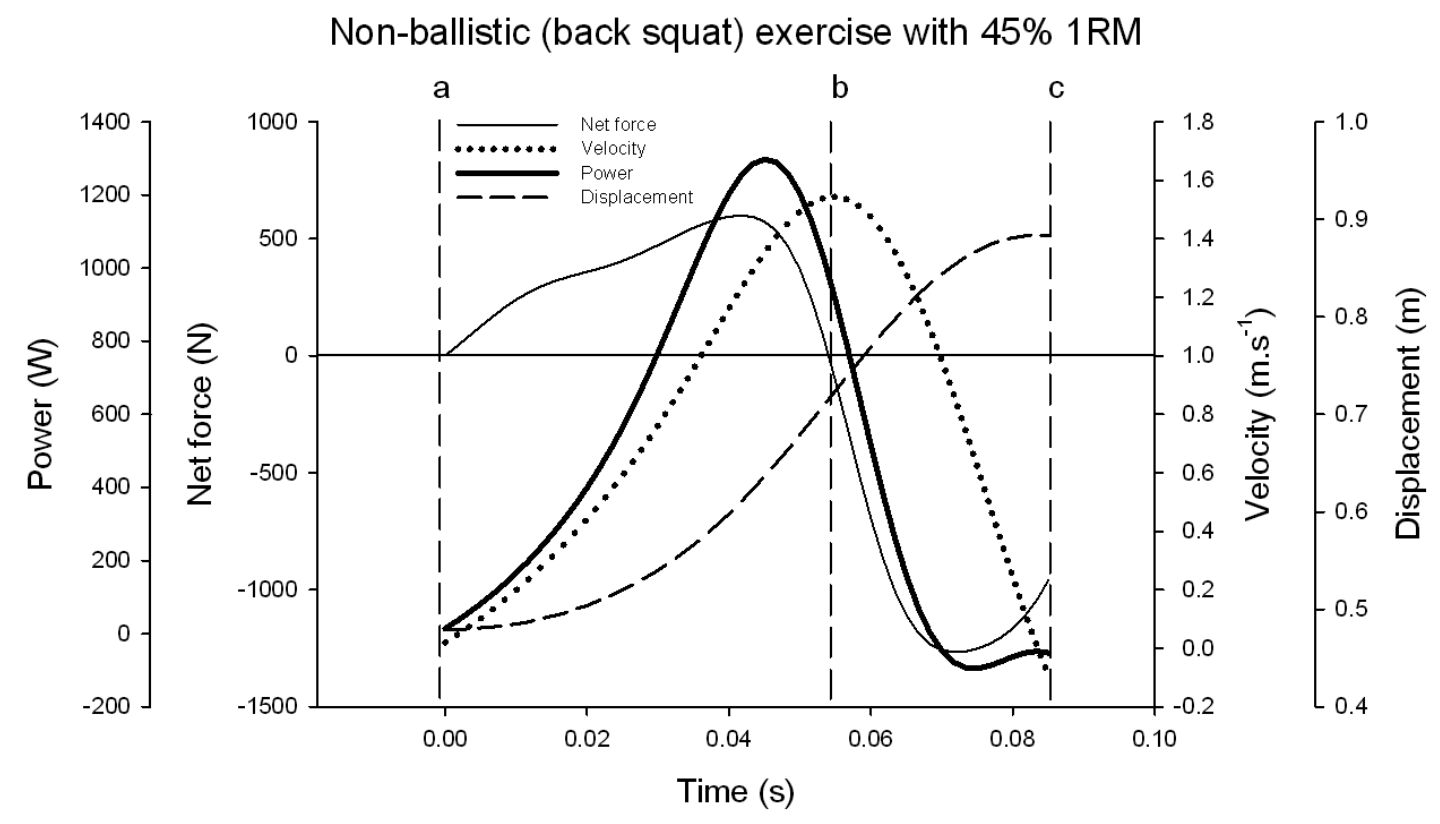

Figure 1 - Determination of the positive lifting phase of nonballistic resistance exercise using the traditional and alternative method. The traditional method begins at the onset of positive barbell displacement (a) ending when maximal barbell displacement is achieved (c) and includes a "braking phase" (Sanchez-Medina et al., 2010) (period between point b and c). The alternative method begins at the same point but only considers positive impulse (point a to point b-excluding the "braking phase").

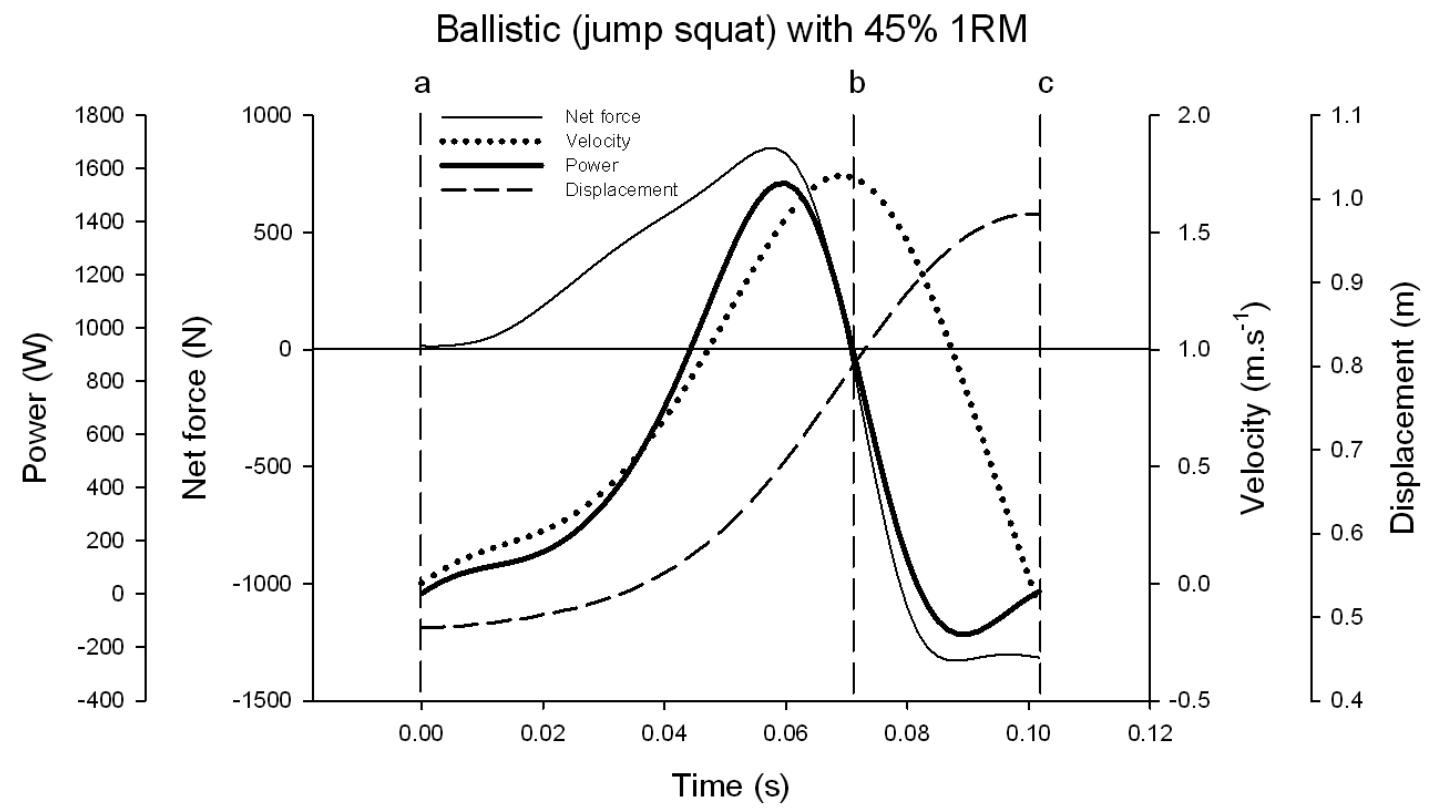

Figure 2 - Determination of the positive lifting phase of ballistic resistance exercise using the traditional and alternative method. The traditional method begins at the onset of positive barbell displacement (a) ending when maximal barbell displacement is achieved (c) and includes a "braking phase" (Sanchez-Medina et al., 2010) (period between point b and c). The alternative method begins at the same point but only considers positive impulse (point a to point b-excluding the "braking phase"). 
Ballistic (jump squat) exercise with 45\% 1RM (including system weight)

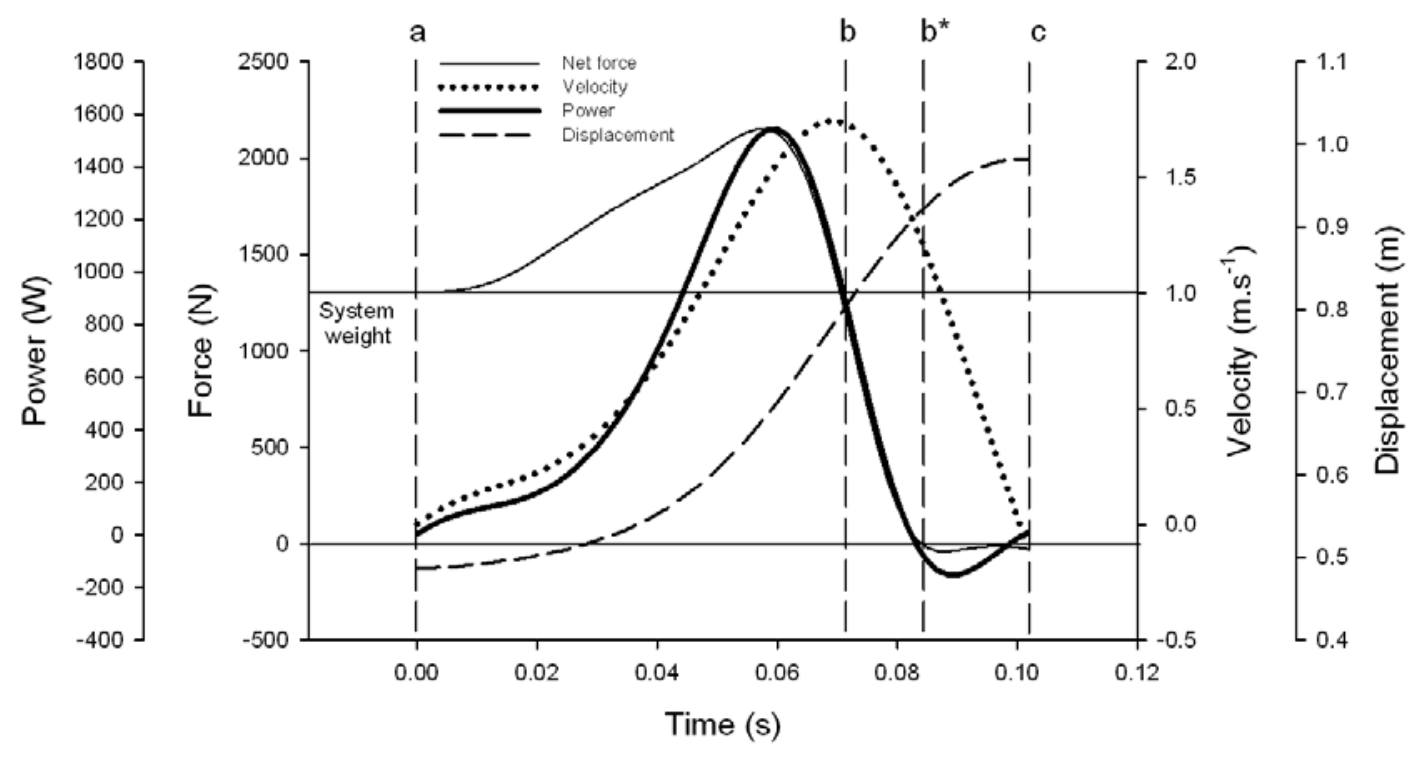

Figure 3 - The way that Frost et al. (2008) applied their alternative method to ballistic resistance exercise including barbell and body system weight (adapted for the lower body). The positive lifting phase begins as above (Figures 1 and 2); system weight is not subtracted but the end of the propulsion phase is still identified as the point at which force decreases to zero (point $b^{*}$ rather than point $b$ ).

failure to only consider net force. It is critical that any new approach proposed for general application is based on sound theoretical principles.

It remains that differences between ballistic and nonballistic lower-body resistance exercise have not been established, although ballistic jump squat exercise is often favored over nonballistic back squat exercise (Cormie et al., 2007; Harris et al., 2008; Hori et al., 2008; Winchester et al., 2008). It also remains unclear whether the method used to identify the positive lifting phase influences any differences that may exist between ballistic and nonballistic lower-body resistance exercise. Therefore, the aims of this study were to establish differences between ballistic and nonballistic lower-body resistance exercise, and to establish whether any differences occurred because of the way the positive lifting phase was determined.

\section{Methods}

\section{Subjects}

Thirty physically active men (mean age $=22.8$ years, $S D$ $=5.5$ years; mean mass $=74.2 \mathrm{~kg}, S D=13.9 \mathrm{~kg}$; mean back squat $1 \mathrm{RM}=130.0 \mathrm{~kg}, S D=34.2 \mathrm{~kg}$ ) with moderate resistance training experience volunteered to participate. All volunteers were required to be fully familiar with both exercises, and be able to perform a modified back squat with a load equivalent to body mass, and were excluded if these criteria were not met. University of Chichester ethics approval was obtained before data collection and following a thorough explanation of the experimental aims and procedures all participants completed a health history questionnaire and provided written informed consent.

\section{Test Procedures}

All participants attended two testing sessions. The first session established maximum strength in a modified back squat (1 RM) seven days before power testing and followed a procedure that was similar to that used by Izquierdo et al. (2002). During back squat exercise, participants squatted until the barbell lightly touched squat rack supports that were set to enable a range of motion that approximated $45 \%$ of the participant's leg length (Flanagan and Salem, 2007), and stood upright to complete the lift. Participants were instructed to perform the descent phase under control and the positive lifting phase as explosively as possible while maintaining foot contact with the ground. The jump squat was performed in the same way as the back squat, but with the aim of jumping from the bottom position for maximum height. The back squat represented nonballistic (NB) exercise, the jump squat, ballistic (B) exercise.

During the second testing session participants performed three sets of three repetitions with 45\% 1RM in each exercise. This load was selected because it represented a compromise between the typical back squat (Izquierdo et al., 2002; Siegel et al., 2002) and jump squat (Harris et al., 2007) optimal loads. The exercise order was counter-balanced with half of the participants performing the back squat first and the other half performing the jump squat exercise first. Participants were instructed to rest for a minimum of one minute and maximum of 
three minutes between each set (measured with a digital stopwatch started when the barbell was racked), with five minutes rest observed between the different exercises (Reiser et al., 1996).

\section{Measurements}

The vertical ground reaction force (barbell and body system force) of B and NB exercise were recorded from both feet individually by two $0.4 \times 0.6 \mathrm{~m}$ Kistler 9851 force platforms (Alton, UK) at a sampling frequency of $500 \mathrm{~Hz}$. Two type 9865E 8-channel charge amplifiers amplified the analog system force signals before they were digitally converted. Two synchronized digital cameras (Basler A602fc-2, Ahrensburg, Germany) were positioned approximately $5 \mathrm{~m}$ from the right-hand end of the barbell with an intercamera angle of about 120 degrees. They filmed a retro-reflective spherical marker that was affixed to and represented the right-hand end of the barbell at $100 \mathrm{~Hz}$ after first recording a 17-point calibration frame (Peak Performance Technologies Inc., Englewood, CO). The marker was digitized for all successful trials at $100 \mathrm{~Hz}$ using Peak Motus 9.2 software. System force and barbell kinematic data collection were synchronized using a Peak event and video control unit (Peak Performance Technologies Inc., Englewood, CO). Digitizing began 10 frames immediately before the bottom position of the lifts, ending 10 frames after maximum barbell displacement. This enabled the calculation of three-dimensional spatial coordinates of the barbell end using the direct linear transformation procedure. Previous research has shown these techniques to be highly reliable, reporting ICC values of between $r=.91$ and 0.95 (Lake et al., 2011).

\section{Data Analysis}

Barbell displacement-time data were filtered using a low-pass second-order Butterworth filter with a cut-off frequency of $6 \mathrm{~Hz}$. Displacement data were then differentiated to determine first velocity then acceleration using the Peak Motus software, and barbell force calculated considering both the acceleration of gravity $(g)$ and the acceleration of the barbell (Hori et al., 2007):

$$
\begin{aligned}
& \text { Barbell force }=(\text { barbell mass } \times g)+ \\
& (\text { barbell mass } \times \text { barbell acceleration })
\end{aligned}
$$

Barbell power output was calculated by multiplying barbell force by barbell velocity. Ground reaction forces recorded from both feet were summed to provide a single measure of system force, and net force was calculated by subtracting barbell and body weight (Kawamori et al., 2005). From this system force, barbell velocity and barbell power were averaged over the traditional and alternative positive lifting phase (see Figure 1). The repetition with the highest mean power output from each of the three sets of three repetitions was selected for analysis (Baker et al., 2001). The durations of the traditional and alternative positive lifting phases were also calculated and from these the relative duration of the positive lifting phase that was spent accelerating the resistance determined from the time taken to achieve peak barbell velocity.

\section{Statistical Analysis}

All data were presented as mean $(S D)$. Differences between $\mathrm{B}$ and NB resistance exercise performance measures, and the influence that the way the positive lifting phase was determined had on the dependent variables, was examined using two-way (exercise $\times$ method) repeated measures analysis of variance. The dependent performance measures of interest were mean force, mean velocity, and mean power. Significant exercise and method effects, and exercise $\times$ method interactions were analyzed using paired sample $t$ tests applying the Bonferroni correction. Differences between $\mathrm{B}$ and NB relative acceleration durations calculated using the peak displacement method were determined using paired sample $t$ tests. The effect that exercise had on force, velocity, power, and relative acceleration duration was also quantified with the effect size $(d)$, using the methods described by Rhea (2004). All statistical calculations were performed using SPSS version 17.0 for Windows (SPSS, Inc., Chicago, IL) and an alpha value of $p \leq .05$ was used to determine statistical significance.

\section{Results}

Descriptive statistics are presented in Table 1. Exercise did not significantly affect mean force $(F=0.645, p=.424)$ or mean power $(F=3.570, p=.061)$, but did significantly affect mean velocity $(F=5.681, p=.019)$. Further analysis showed that B mean velocity was significantly greater than NB mean velocity $(14 \%, p=.019)$; however, the mean velocity effect size was very low $(d=0.110)$.

Method did not significantly affect mean velocity $(F=0.816, p=.368)$, but did significantly affect mean force $(F=91.175, p<.0001)$, and mean power $(F=$ $17.736, p<.0001)$. Further analysis showed that positive impulse mean force was significantly greater than peak displacement mean force $(24 \%, p<.0001)$, and that positive impulse mean power was significantly greater than peak displacement mean power $(29 \%, p<.0001)$. However, the mean force effect size was moderately low $(d=0.440)$, and the mean power effect size very low $(d=0.199)$.

The results of the $t$ test showed that $\mathrm{B}$ relative acceleration duration was $8.6 \%$ greater than $\mathrm{NB}$ relative exercise duration $(t=3.109, p=.004, d=0.86)$ when peak displacement was used to identify the end of the positive lifting phase.

\section{Discussion}

The aim of this study was to establish differences between $\mathrm{B}$ and NB lower-body resistance exercise, and different methods of determining their positive lifting phase. 
Table 1 Mean (SD) and effect sizes (d) B and NB positive lifting phase force, velocity, power, and relative acceleration durations

\begin{tabular}{|c|c|c|c|c|c|c|c|c|c|c|c|c|}
\hline & \multicolumn{2}{|c|}{ Force (N) } & \multirow[b]{2}{*}{$d$} & \multicolumn{2}{|c|}{$\begin{array}{c}\text { Velocity } \\
\left(\mathrm{m} \cdot \mathrm{s}^{-1}\right)\end{array}$} & \multirow[b]{2}{*}{$d$} & \multicolumn{2}{|c|}{ Power (W) } & \multirow[b]{2}{*}{$d$} & \multicolumn{2}{|c|}{$\begin{array}{c}\text { Relative } \\
\text { Acceleration } \\
\text { Duration (\%) }\end{array}$} & \multirow[b]{2}{*}{$d$} \\
\hline & B & NB & & B & NB & & B & NB & & B & NB & \\
\hline & 1330.96 & 1324.36 & & 1.05 & 0.89 & & 613.73 & 522.42 & & 64 & 70 & \\
\hline \multirow[t]{3}{*}{ PD } & & & 0.03 & & & 0.48 & & & 0.46 & & & 0.86 \\
\hline & (207.14) & (216.51) & & $(0.33)$ & $(0.33)$ & & (202.59) & (197.49) & & (10) & (4) & \\
\hline & 1768.27 & 1706.00 & & 0.98 & 0.86 & & 855.94 & 740.67 & & & & \\
\hline PI & & & 0.24 & & & 0.37 & & & 0.31 & 100 & 100 & N/A \\
\hline & $(260.85)$ & $(250.71)$ & & $(0.32)$ & $(0.33)$ & & $(374.03)$ & $(372.36)$ & & & & \\
\hline
\end{tabular}

Note. $\mathrm{B}=$ ballistic; $\mathrm{NB}=$ nonballistic; $\mathrm{PD}=$ peak displacement (point c, Figure 1); PI = positive impulse (point b, Figure 1).

Regarding mean positive lifting phase force, the results of this study demonstrated that NB performance was underpinned by the same mechanical demands as B performance. This does not agree with earlier research that considered B and NB upper-body resistance exercise using the peak displacement method of determining the positive lifting phase (Frost et al., 2008; Newton et al., 1996), but agrees with research that considered positive lifting phases determined using the positive impulse method (Frost et al., 2008). Frost et al. (2008) suggested that mean positive lifting phase force is the same during maximal effort NB and B performance, although performance outcomes from the current study present an obvious difference in terms of significantly different velocities. This may occur because the necessity to arrest barbell displacement during NB resistance exercise underpins differences in NB and B mean positive lifting phase velocity, because at the conclusion of the NB propulsion phase negative work is performed as the lifter applies forces in a direction that opposes barbell displacement. This is not the case during B lower-body resistance exercise performance, where the lifter aims to jump as high as possible.

However, differences in velocity did not carry over to mean power. Although mean B power was $14 \%$ greater than mean NB power (regardless of method used to determine the positive lifting phase) variance was large. This was clearly demonstrated by moderate effect sizes that did not exceed $d=0.46$. Therefore, the popular contention of B lower-body resistance exercise superiority (Newton et al., 1996) may be unfounded. However, further research considering a range of different loads and the effects of $B$ and NB lower-body resistance training intervention needs to be performed before the contention of B lower-body resistance exercise superiority can be ruled out.

It is important to note the limitations of the alternative method of determining the positive lifting phase that was proposed by Frost et al. (2008). The exclusion of the braking phase enables a theoretically more robust method of identifying mechanical demands of B and NB resistance exercise because the positive or propulsion impulse is common to both exercise types. However, theoretical integrity can only be achieved if basic mechanical principles are observed. It is important that strength and conditioning practitioners and investigators have a sound understanding of the differences between the methods used in this study to determine the positive lifting phase. Further, it is critical that the positive impulse method of determining the positive lifting phase uses net force, remembering that net force is obtained by subtracting barbell and body system weight from absolute force (Driss et al., 2001; Kawamori et al., 2005).

Only net forces exceeding system weight influence barbell and body system center of mass kinematics. If ground reaction force is recorded using a force platform this applies to the barbell and body system center of mass. If force is derived from barbell kinematics, net barbell force should be considered (subtract barbell weight), especially if the method proposed by Hori et al. (2007) is used to derive barbell force from kinematic measures.

Further, strength and conditioning practitioners and investigators must understand that when net force (ground reaction or barbell) decreases below zero $\mathrm{N}$ a number of factors can be observed. First, regardless of whether the exercise of interest is $\mathrm{B}$ or $\mathrm{NB}$, this point marks the end of resistance acceleration (whether barbell or system center of mass); second, all displacement of the resistance of interest past this point is an expression of momentum; and third, this point coincides with peak resistance velocity. However, if barbell and body system force is used to determine the positive impulse then the peak of the system center of mass velocity derived from net force will coincide with this point. If net force is derived from barbell kinematics, the end of the barbell positive impulse will correspond with peak barbell velocity. This could be practically applicable to strength and conditioning practitioners who do not have access to a force platform but can access basic motion analysis 
systems, as the identification of peak barbell velocity will enable the relatively simple, but more theoretically robust way of determining the propulsion phase of the positive lifting phase. Review of the literature indicates that this method has been used before to identify the end point of the snatch and clean second pull propulsion phase (Garhammer, 1991).

Although the results of this study could have important implications for strength and conditioning practitioners there were several methodological limitations that must considered. Firstly, only one load was considered, and while this load represented a compromise between the loads that typically maximize both B (30\% 1RM; Harris et al., 2007) and NB (60\% 1RM; Izquierdo et al., 2002; Siegel et al., 2002) power, it is possible that any reported differences (or lack thereof) may differ for lighter or heavier loads. Secondly, to control exercise range of motion participants were instructed to squat until they lightly touched the safety support of the squat rack supports before positive lifting phase was performed. This may have limited the potential to use elastic energy generated during the descent phase, particularly during $\mathrm{B}$ performance, and therefore $\mathrm{B}$ and NB resistance exercise performance.

To summarize, B and NB lower-body resistance exercise mean force and power were not significantly different, but B and NB lower-body resistance exercise mean velocity was. Further, the method used to determine the positive lifting phase significantly affects mean force, velocity, and power, but does not significantly affect differences between B and NB lower-body resistance exercise. This is an area that requires further research attention, but the findings suggest that strength and conditioning coaches may need to review the common perception that B lower-body resistance exercise is superior for developing lower-body power.

\section{References}

Baker, D., Nance, S., \& Moore, M. (2001). The load that maximizes the average mechanical power output during jump squats in power-trained athletes. Journal of Strength and Conditioning Research, 15, 92-97. PubMed

Cormie, P., McCaulley, G.O., \& McBride, J.M. (2007). Power versus strength-power jump squat training: Influence on the load-power relationship. Medicine and Science in Sports and Exercise, 39, 996-1003. PubMed

Dowling, J.J., \& Vamos, L. (1993). Identification of kinetic and temporal factors related to vertical jump performance. Journal of Applied Biomechanics, 9, 95-110.

Driss, T., Vandewalle, H., Quievre, J., Miller, C., \& Monod, H. (2001). Effects of external loading on power output in a squat jump on a force platform: A comparison between strength and power athletes and sedentary individuals. Journal of Sports Sciences, 19, 99-105. PubMed doi:10.1080/026404101300036271

Flanagan, S., \& Salem, G. (2007). Bilateral differences in the net joint torques during the squat exercise. Journal of Strength and Conditioning Research, 21, 1220-1226. PubMed

Frost, D.M., Cronin, J.B., \& Newton, R.U. (2008). Have we underestimated the kinematic and kinetic benefits of non-ballistic motion? Sports Biomechanics, 7, 372-385. PubMed doi:10.1080/14763140802273005

Garhammer, J. (1991). A comparison of maximal Power outputs between elite male and female weightlifters in competition. Journal of Applied Biomechanics, 7, 3-11.

Gonzalez-Badillo, J.J., \& Sanchez-Medina, L. (2010). Movement velocity as a measure of loading intensity in resistance training. International Journal of Sports Medicine, 31, 347-352. PubMed doi:10.1055/s-0030-1248333

Harman, E.A., Rosenstein, M.T., Frykman, P.N., Rosenstein, R.M., \& Kraemer, W.J. (1991). Estimation of human power output from vertical jump. Journal of Applied Sports Science Research, 5, 116-120.

Harris, N.K., Cronin, J.B., \& Hopkins, W.G. (2007). Power outputs of a machine squat-jump across a spectrum of loads. Journal of Strength and Conditioning Research, 21, 1260-1264. PubMed

Harris, N.K., Cronin, J.B., Hopkins, W.G., \& Hansen, K.G. (2008). Squat jump training at maximal power loads vs. heavy loads: Effect on sprint ability. Journal of Strength and Conditioning Research, 22, 1742-1749. PubMed doi:10.1519/JSC.0b013e318187458a

Hori, N., Newton, R.U., Andrews, W.A., Kawamori, N., McGuigan, M.R., \& Nosaka, K. (2007). Comparison of four different methods to measure power output during the hang power clean and the weighted jump squat. Journal of Strength and Conditioning Research, 21, 314-320. PubMed

Hori, N., Newton, R.U., Kawamori, N., McGuigan, M.R., Andrews, W.A., Chapman, D.W., \& Nosaka, K. (2008). Comparison of weighted jump squat training with and without eccentric braking. Journal of Strength and Conditioning Research, 22, 54-65. PubMed doi:10.1519/ JSC.0b013e31815ef052

Izquierdo, M.H., Hakkinen, K., Gonzalez-Badillo, J.J., Ibanez, J., \& Gorostiaga, E.M. (2002). Effects of long-term training specificity on maximal strength and power of the upper and lower extremities in athletes from different sports. European Journal of Applied Physiology, 87, 264-271. PubMed doi:10.1007/s00421-002-0628-y

Jandacka, D., \& Vaverka, F. (2008). A regression model to determine load for maximum power output. Sports Biomechanics, 7, 361-371. PubMed doi:10.1080/14763140802266934

Kawamori, N., Crum, A.J., Blumert, P.A., Kulik, J.R., Childers, J.T., Wood, J.A., . . Haff, G.G. (2005). Influence of different relative intensities on power output during the hang power clean: Identification of the optimal load. Journal of Strength and Conditioning Research, 19, 698-708. PubMed

Lake, J., Lauder, M., \& Smith, N. (2011). Does side dominance affect the symmetry of barbell end kinematics during lower-body resistance exercise? Journal of Strength and Conditioning Research, 25, 872-878. PubMed

Linthorne, N.P. (2001). Analysis of standing vertical jumps using a force platform. American Journal of Physics, 69, 1198-1204. doi:10.1119/1.1397460

Newton, R.U., Kraemer, W.J., Hakkinen, K., Humphries, B.J., \& Murphy, A.J. (1996). Kinematics, kinetics and muscle activation during explosive upper-body movements. Journal of Applied Biomechanics, 12, 31-43.

Reiser, R.F., Smith, S.L., \& Rattan, R. (1996). Science and technology to enhance weightlifting performance: The Olympic program. Strength and Conditioning Journal, 18, 43-51. doi:10.1519/1073-6840(1996)018<0043:SAT TEW $>2.3 . \mathrm{CO} ; 2$ 
Rhea, M.R. (2004). Determining the magnitude of treatment effects in strength training research through the use of the effect size. Journal of Strength and Conditioning Research, 18, 918-920. PubMed

Sanchez-Medina, L., Perez, C.E., \& Gonzalez-Badillo, J.J. (2010). Importance of the propulsive phase in strength assessment. International Journal of Sports Medicine, 31, 123-129. PubMed doi:10.1055/s-0029-1242815

Siegel, J.A., Gilders, R.M., Staron, R.S., \& Hagerman, F.C. (2002). Human muscle power output during upper and lower-body exercises. Journal of Strength and Conditioning Research, 16, 173-178. PubMed

Winchester, J., McBride, J., Maher, M., Mikat, R., Allen, B., Kilne, D., \& McGuigan, M. (2008). Eight weeks of ballistic exercise improves power independently of changes in strength and muscle fibre type expression. Journal of Strength and Conditioning Research, 22, 1728-1734. PubMed doi:10.1519/JSC.0b013e3181821abb 
Copyright of Journal of Applied Biomechanics is the property of Human Kinetics Publishers, Inc. and its content may not be copied or emailed to multiple sites or posted to a listserv without the copyright holder's express written permission. However, users may print, download, or email articles for individual use. 
Copyright of Journal of Applied Biomechanics is the property of Human Kinetics Publishers, Inc. and its content may not be copied or emailed to multiple sites or posted to a listserv without the copyright holder's express written permission. However, users may print, download, or email articles for individual use. 